OPEN ACCESS

Edited by:

Enrico Baruffini,

University of Parma, Italy

Reviewed by:

Florence Fellmann,

Lausanne University Hospital,

Switzerland

Maria Paola Lombardi, University of Amsterdam, Netherlands

Federica Sangiuolo,

University of Rome Tor Vergata, Italy

*Correspondence:

Jernej Kovač

jernej.kovac@kclj.si

Specialty section: This article was submitted to

Genetic Disorders,

a section of the journal

Frontiers in Genetics

Received: 17 March 2017

Accepted: 21 June 2017

Published: 30 June 2017

Citation:

Kovač J, Klančar G,

Trebušak Podkrajšek K and

Battelino S (2017) Discovering the Unexpected with the Utilization

of NGS in Diagnostics

of Non-syndromic Hearing Loss

Disorders: The Family Case of ILDR1-Dependent Hearing Loss

Disorder. Front. Genet. 8:95.

doi: 10.3389/fgene.2017.00095

\section{Discovering the Unexpected with the Utilization of NGS in Diagnostics of Non-syndromic Hearing Loss Disorders: The Family Case of ILDR1-Dependent Hearing Loss Disorder}

\author{
Jernej Kovač ${ }^{*}$, Gašper Klančar ${ }^{1}$, Katarina Trebušak Podkrajšek ${ }^{1,2}$ and Saba Battelino ${ }^{2,3}$ \\ 1 Unit of Special Laboratory Diagnostics, University Children's Hospital, University Medical Centre Ljubljana, Ljubljana, \\ Slovenia, ${ }^{2}$ Faculty of Medicine, University of Ljubljana, Ljubljana, Slovenia, ${ }^{3}$ Department of Otorhinolaryngology and \\ Cervicofacial Surgery, University Medical Centre Ljubljana, Ljubljana, Slovenia
}

Sensorineural hearing loss (SNHL) is a heterogeneous family of hearing disabilities with congenital (including genetic) as well as acquired etiology. Congenital SNHL of genetic etiology is further sub-divided into autosomal dominant, autosomal recessive and $\mathrm{X}$-linked SNHL. More than 60 genes are involved in the etiology of autosomal recessive non-syndromic hearing loss (ARNSHL) commonly manifesting as heterogeneous prelingual profound to severe non-progressive clinical phenotype. ILDR1-dependent ARNSHL (DFNB42, OMIM: \# 609646) is a very rare sub-type of hearing disability, with unknown prevalence, caused by function-damaging genetic variants in ILDR1 gene reported in families of Middle-Eastern origin. ILDR1 (Immunoglobulin-Like Domaincontaining Receptor 1) is involved in the development of semicircular canal, tricellular tight junction and auditory hair cells. An apparently non-consanguineous family of European ancestry with two affected siblings with profound progressive hearing loss characterized in their infancy and successfully treated with cochlear implants $(\mathrm{Cl})$ is presented. Genetic analysis of common ARNSHL genetic causes in the population of origin was negative, thus the next-generation sequencing (NGS) and family segregation analysis to identify underlying causative genetic variant was performed. Unexpectedly and atypical for the population of origin a homozygous non-sense variant ILDR1 c.942C > A (p.Cys314Ter) inherited from both heterozygous parents was identified in both patients. Contrary to the commonly reported phenotype, indices of a progressive hearing loss and potential compensatory mechanism of vestibular function were revealed with the analysis of clinical data. The utilization of NGS was demonstrated as an invaluable tool for the detection of atypical rare variants in diagnostics of unidentified hearing loss disorders.

Keywords: ILDR1, hearing loss, cochlear implant, orphan disease, NGS analysis 


\section{CASE PRESENTATION}

A family case of hereditary hearing loss in two siblings of apparently non-consanguineous parents of European ancestry is presented. The evaluation protocol assessed detailed family history and medical history with focus on potential causes of acquired hearing loss (acoustic trauma, intrauterine infections, perinatal complications, meningitis, mumps, and prenatal/postnatal ototoxic drug exposure). Additionally, a complete audiological history was recorded to establish the age of the hearing loss onset, the rate of hearing loss progression and to identify other audiological symptoms. Both index patients and their parents underwent a clinical otorhinolaryngological examination, including ear microscopy, with a systematic search for syndromic hearing loss indices. A tympanogram was performed and middle-ear associated causes of hearing loss were excluded. Additionally, they underwent ophthalmological and pedo-neurological examinations. The conditional and partly conditioned classical pure tone audiogram (PTA) was recorded in speech frequencies $0.25,0.5,1,2$, and $4 \mathrm{kHz}$, respectively, followed by the brainstem evoked response audiometry (BERA) analysis with up to $110 \mathrm{~dB}$ click stimulation. The function of vestibular organs was tested with caloric stimulation by irrigation of the external ear canal with $50 \mathrm{~cm}^{3}$ of water at 44 , 30 , and $17^{\circ} \mathrm{C}$, respectively. Before cochlear implant surgery (CI) the computed tomography (CT scan) of the temporal bone was performed to evaluate the anatomy of medial and inner ear. Additionally, cochleography and electrically evoked auditory brain responds (EABR) test by reversed electrical $200 \mu$ s long stimuli with intensity range from $200 \mu \mathrm{A}$ to $1 \mathrm{~mA}$, using stimulating golf electrode placed on a round window was performed. This study was carried out in accordance with the recommendations of the Declaration of Helsinki and the Slovenian national medical ethics committee. All subjects or their legal guardians gave written informed consent in accordance with the Declaration of Helsinki. The protocol was approved by the Slovenian national medical ethics committee (\#34/4/07).

First sibling (II,2; age range: 35-40 years) was admitted for the first time to an audiologist (age range: $0-5$ years) due to hearing disorder and fitted with bilateral hearing aids. The caloric stimulation of both vestibular organs was negative, yet the patient did not present with any vestibular problems in recorded medical history. Interestingly, the speech was well developed, enabling education by regular educational program. The hearing above $3 \mathrm{kHz}$ rapidly deteriorated, followed by relatively stable period until the adulthood (age range: 30-35 years) when only some islands of residual hearing were detected at lower frequencies. The level of hearing perception on PTA test performed at the first audiologist examination were $50,70,85,100$, and $100 \mathrm{~dB}$ for the right side and $40,55,70,90$, and $90 \mathrm{~dB}$ for left side, respectively. Later the hearing significantly worsened with PTA thresholds reaching $90,105,110,115$, and $120 \mathrm{~dB}$ at the right side and 85 , $100,110,115$, and $115 \mathrm{~dB}$ at the left side for stimulation at 0.25 , $0.5,1,2$, and $4 \mathrm{kHz}$ frequencies, respectively (Figure 1A). The computed tomography (CT scan) of the temporal bone revealed normal medial and inner ear. Surprisingly, contrary to the older

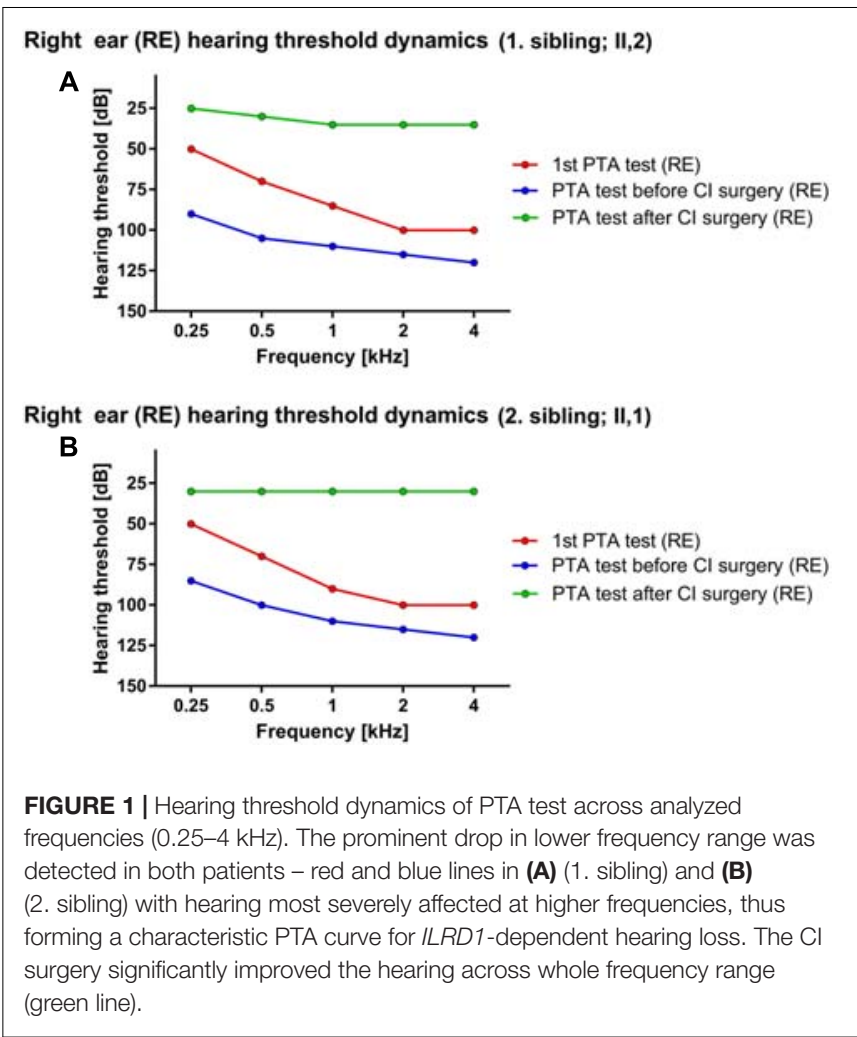

results the classical bi-thermal caloric stimulation with water at 30 and $44^{\circ} \mathrm{C}$ at adulthood (age range: $30-35$ years) revealed symmetrical, good responses. The cochleography measurements were negative where EABR showed responses at stimulation with $1 \mathrm{~mA}$ on the right side. Consequently, the patient received CI on the right side and after the CI surgery, the PTA of implanted side reached between 25 and $35 \mathrm{~dB}$ across tested frequencies.

Due to a family history of hearing loss the second patient (II,1; age range: $35-40$ years; a sibling to the index patient) was sent to an audiologist soon after birth (age range: 0-1 year). Comparatively, the PTA test in the childhood (age range: $0-5$ years) revealed the hearing thresholds at 50,70,90,100, and $100 \mathrm{~dB}$ for the right ear and $50,65,80,90$, and $110 \mathrm{~dB}$ on the left side at $0.25,0.5,1,2$, and $4 \mathrm{kHz}$ frequency range, respectively. The hearing progressively deteriorated in the adulthood (age range: 25-30 years) the PTA thresholds dropped to $85,100,110,115$, and $120 \mathrm{~dB}$ for the right side and $85,95,120,120$, and $120 \mathrm{~dB}$ for the left side at $0.25,0.5,1,2$, and $4 \mathrm{kHz}$ frequency range, respectively (Figure 1B). The BERA potentials were not detected even at stimulation with $110 \mathrm{~dB}$ click. On the other hand, the response of vestibular organs to caloric stimulation was well detected and symmetrical. The patient was fitted with bilateral hearing aids at the first year of age. The speech development was not hindered and the second patient completed regular educational program. The hearing further deteriorated to the level where no benefit from conventional hearing aids was detected (age range: 25-30 years). The CT scan of temporal bones was normal. The cochleography measurements were negative where the EABR 
showed responses at stimulation with $300 \mu \mathrm{A}$ on the right side and at $400 \mu \mathrm{A}$ on the left side. After CI (age range: $25-30$ years) the PTA on implanted side detected the hearing level of $30 \mathrm{~dB}$ through the whole frequency range.

No syndromic hearing loss indices or middle-ear related causes of hearing loss were identified in both patients. Air-bone gap did not exceed $5 \mathrm{~dB}$. Additionally, both parents had normal hearing and all possible known external causes of hearing loss were excluded in both siblings.

Whole blood EDTA samples were collected for isolation of genomic DNA according to established laboratory protocols with FlexiGene DNA isolation kit (Qiagen, Hilden, Germany) (Kovač et al., 2014), to identify the underlying genetic cause of hearing loss in both siblings. Testing for the most common genetic causes of hearing loss in the population of origin the GJB2 and TMPRSS3 variants (Battelino et al., 2015), was negative, thus qualifying both siblings for next-generation sequencing (NGS) analysis. The NGS library was prepared using TruSight One sequencing panel (Illumina, San Diego, CA, United States) according to manufacturer's instructions. The loading concentration of prepared NGS library was $12 \mathrm{pM}$. MiSeq desktop sequencer together with MiSeq Reagent kit v3 (both Illumina, San Diego, CA, United States) were used for data collection followed by on-board primary analysis. Genetic variants with coverage $>15 \times$ were analyzed with Variant Studio 2.2 software (Illumina, San Diego, CA, United States). All coding variants located outside of 100 genes related to hearing loss, reported in Hereditary Hearing Loss Homepage, were excluded from further analysis. The minor allele frequency threshold for known variants was set at $1 \%$ and all variants exceeding this value were excluded from further analysis as well.

The NGS analysis revealed that both siblings were carriers of homozygous ILDR1 non-sense mutation c.942C > A (NM_001199799.1), introducing early stop codon at Cys314. The coverage of identified variant was $189 \times$ and $126 \times$ in first and second sibling, respectively. Sanger sequencing confirmed the presence of the genetic variant and its zygosity. Additionally, the family segregation analysis was performed by targeted Sanger sequencing of parental DNA samples. Both parents were carriers of a heterozygous ILDR1 c.942C $>$ A variant (Figure 2).

Additional haplotype analysis of low-coverage mitochondrial genome, extracted from NGS data, was performed to obtain the insight in the ancestry of the family. Using Integrative Genomics Viewer (Thorvaldsdóttir et al., 2013) and Mitomaster (Lotti et al., 2013) tools, the mitochondrial genome haplotype of both patients was classified as J1c3a2. The identification of Y-chromosome haplotype was not successful due to too low Y-chromosome coverage in acquired NGS data.

Moreover, the ratio between rare (MAF $<1 \%$ ) homozygous and heterozygous genetic variants (rare hom/het ratio) was calculated as a proxy to further assess the level of consanguinity in the family. The corresponding standard deviation score (SDS) of rare hom/het ratio was derived from comparison of patient's ratios to a distribution of rare hom/het ratio in the Slovenian population. The population distribution of rare hom/het ratio was calculated from sequencing data of 117 anonymized individuals, generated during a routine genetic diagnostic procedure using identical abovementioned NGS library preparation protocol (TruSight One sequencing panel and MiSeq Reagent kit v3, both Illumina, San Diego, CA, United States). The coverage threshold for all variants' datasets was set to $>20 \times$, and only variants passing this threshold were taken into a calculation of SDS of rare hom/het ratio. The SDS of rare hom/het ratio was 2.66 for the first sibling (II,2) and 1.42 for the second sibling (II,1).

\section{BACKGROUND}

Sensorineural hearing loss (SNHL) is a family of heterogeneous conditions spanning acquired as well as congenital causes of the disease. Genetic changes are one of the main contributing factors in the development of congenital SNHL. Non-syndromic hearing loss of genetic etiology has more than 100 genetic loci involved in its etiology (Parker and Bitner-Glindzicz, 2015). Additionally, more than 60 genes (Van Camp and Smith, 2017) are involved in the etiology of autosomal recessive non-syndromic hearing loss (ARNSHL), which phenotype is usually profound to severe, non-progressive and pre-lingual (Petersen and Willems, 2006). The most common genetic cause of ARNSHL are GJB2 mutations while other reported genes include SLC26A4, MYO7A, OTOF, CDH23 and TMC1. Interestingly, GJB2 mutations are responsible for approximately $27 \%$ and TMPRSS3 mutations are responsible for around $13 \%$ of remaining Slovenian ARNSHL population (Battelino et al., 2015). The advent of next generation sequencing and its introduction into routine genetic diagnostic procedures accelerated the identification of causative genetic variants across heterogeneous population of patients with SNHL (Diaz-Horta et al., 2012; Yan et al., 2013).

Loss-of-function ILDR1 mutations have been implicated in the development of very rare non-syndromic autosomal recessive deafness type 42 (DFNB42) in humans (Borck et al., 2011). ILDR1 gene encoding the immunoglobulin-like domain containing receptor 1 is associated with the development of semicircular canal, tricellular tight junction and auditory hair cells in zebrafish and mouse models (Higashi et al., 2013; Morozko et al., 2014; Sang et al., 2014). Moreover the functional characterization of identified ILDR1 variants in mouse mammary epithelial EpH4 cell lines revealed the disruption of tricellulin (a component of tricellular tight junction) recruitment by ILDR1 and failure to form tight junction (Kim et al., 2015).

\section{DISCUSSION}

A detailed clinical phenotype of two siblings with subsequently identified loss-of-function ILDR1 mutation, from a family of European ancestry with profound hearing loss, treated with CI, is presented (Figure 2). The non-sense homozygous mutation in ILDR1 gene inherited from both parents was identified as the cause of their disability. Classified as DFNB42 hearing loss, it is a very rare disorder reported in families of Pakistani, Saudi Arabian, Turkish and Iranian origin (Borck et al., 2011; Ramzan 


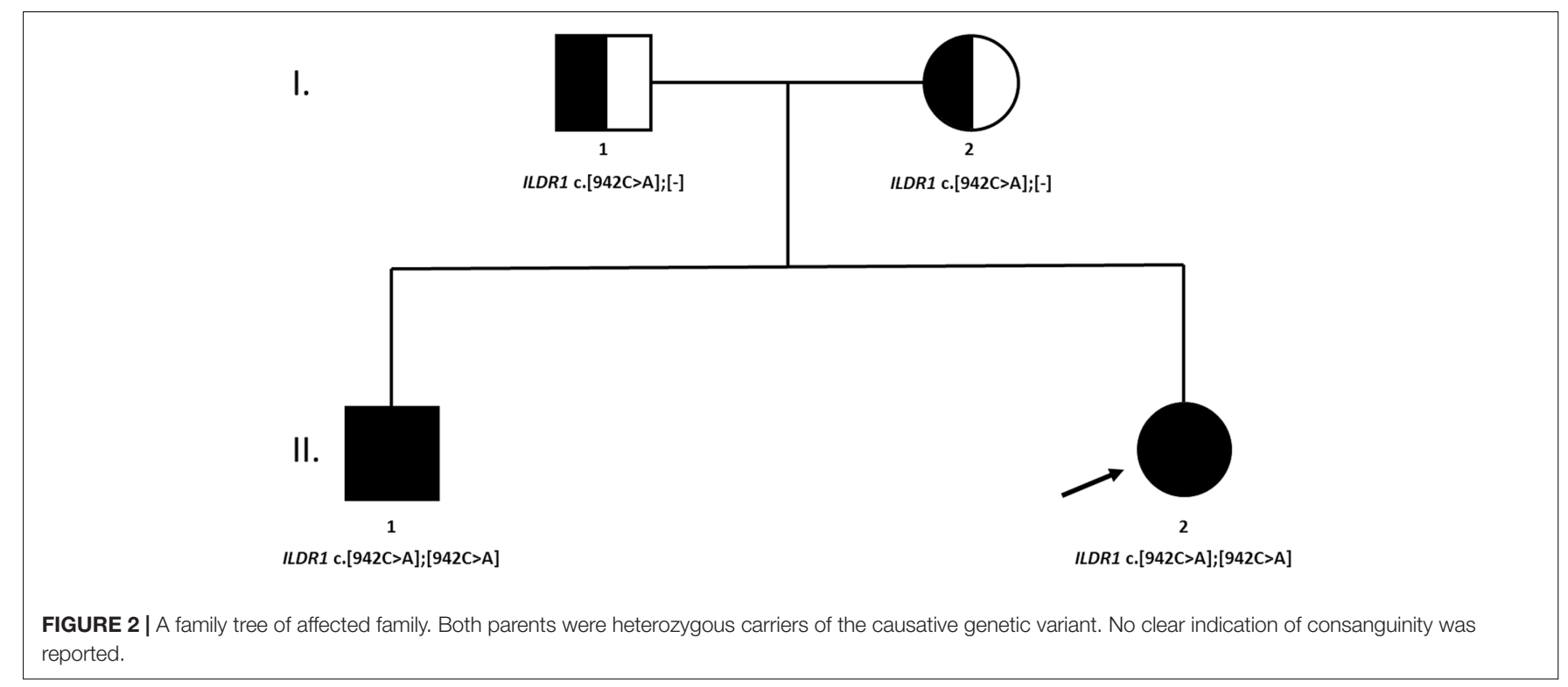

et al., 2014; Bademci et al., 2015; Mehrjoo et al., 2015) and to our knowledge this is the first report of its occurrence in the European population. The identified genetic variant ILDR1 c.942C $>$ A introduces early stop codon p.Cys314Ter and is reported in Human Genome Mutation Database as a disease causing variant (CM163808). It probably renders the ability of ILDR 1 to recruit tricellulin and effectively form tight junctions inefficient, consequently affecting the function of auditory hair cells as shown in animal models and cellular cultures (Morozko et al., 2014; Sang et al., 2014; Kim et al., 2015). Genetic variant ILDR1 c.942C > A was previously reported as causative in three families of Turkish origin indicating the potential Middle-Eastern origin of the mutation (Bademci et al., 2015). The minor allele frequency of identified variant in Exome Aggregation Consortium database $^{1}$ is $1 / 60642$ classifying it as a very rare variant.

Analyzing the PTA data, the characteristic curves with more prominent hearing loss in higher frequency range were revealed (Figure 1) coinciding with previous reports (Kim et al., 2015) although there are some reports where "flat" audiograms are reported as well (Borck et al., 2011) indicating potentially diverse clinical phenotype of DFNB42. Moreover, contrary to the commonly reported phenotype (Borck et al., 2011) both patients presented with progressive and post-lingual hearing loss with the most rapid progression in the lower frequency range (Figure 1), supporting phenotype diversity of DFNB42 clinical manifestation. Additionally, a recent report on ILDR1-knockout mouse models demonstrated the progressive degradation of outer hair cells and organ of Corti, further supporting the progressive hearing loss disorder phenotype (Aslam et al., 2005; Sang et al., 2015). Nevertheless, both patients successfully finished their education program benefiting from the support of hearing aids and CI and successfully established their professional careers thereafter. The CI rescued the hearing of patients, restoring

${ }^{1}$ http://exac.broadinstitute.org hearing in whole frequency range (Figure 1) to approximately $30 \mathrm{~dB}$ and drastically improved patients' quality of life although there is a report where CI in ILDR1-misense-mutation dependent hearing loss was not successful (Kim et al., 2015). Nevertheless, when performed without clinical complications the CI is the clinical procedure of choice for optimal recovery of hearing in patients with ILDR1-dependent hearing loss. Additionally, the potential recovery of vestibular activation in the first patient was noticed. First caloric stimulation test of vestibular organ at the age of three was negative but the repeated test before the CI (27 years later) revealed that the vestibular response recovered. The medical records were carefully reanalyzed and the possibility of a potential analytical error was excluded. Although the existence of potential underlying recovery mechanism is a speculation, there are reports, which indicate that, at least in mice, the recovery of vestibular function could be guided through ILDR1-loss-of-function dependent angulin-1 mediated recovery of tricellulin localization. However, tricellulin/angulin-1 interaction does not recover hearing in mice and consequently the potential positive effect on vestibular function has to be further investigated (Higashi et al., 2015).

Consanguinity is a commonly reported underlying characteristic of ILDR1-dependent hearing loss resulting in a homozygous function-damaging variant segregating in affected family members (Borck et al., 2011; Ramzan et al., 2014), nevertheless, the self-reported data regarding the consanguinity in the a patient's family didn't indicate its possibility. But relatively high SDS of rare hom/het ratio (2.66 and 1.42 , respectively) implies that at least a distant consanguinity may be a reasonable assumption. The difference between SDS of both siblings may look relatively distinct, but it translates in to the nominal difference of $1.93 \%$ ( $8.29 \%$ vs. $6.34 \%$, respectively). The inter-sibling discrepancy of rare hom/het ratio SDS may originate from NGS data and library preparation alone, as both samples were not sequenced with the same quality and coverage of specific genomic regions. Consequently, this empirical 
evaluation may not be very precise and should be taken with a degree of reticence to use it as definite indicator of consanguinity. More accurate mapping of parental homozygous regions could not be performed as parents gave consent only to be tested for identified causative genetic variant in ILDR1 gene. The haplotype analysis of low-coverage mtDNA revealed that both siblings are carriers of J1c3a2 haplotype. The mitochondrial haplogroup J is widespread in Europe as well as Near East. It is assumed that it was introduced into European population through Neolithic and/or Late Glacial migrations (Pala et al., 2012). Consequently, the origin of the family was established only on self-reported data from both patients and their parents.

\section{CONCLUDING REMARKS}

This analysis confirmed the underlying variability of clinical phenotype in ILDR1-dependent hearing loss (Kim et al., 2015), and additionally supported the possibility of DFNB42 being a progressive hearing loss disorder (Kim et al., 2015; Sang et al., 2015), successfully treatable by CI. Additionally, this case clearly demonstrated the advantage of NGS technology in diagnostic algorithms of orphan diseases such as DFNB42. Using targeted Sanger sequencing and following the reductionist

\section{REFERENCES}

Aslam, M., Wajid, M., Chahrour, M. H., Ansar, M., Haque, S., Pham, T. L., et al. (2005). A novel autosomal recessive nonsyndromic hearing impairment locus (DFNB42) maps to chromosome 3q13.31-q22.3. Am. J. Med. Genet. A 133A, 18-22. doi: 10.1002/ajmg.a.30508

Bademci, G., Foster, J., Mahdieh, N., Bonyadi, M., Duman, D., Cengiz, F. B., et al. (2015). Comprehensive analysis via exome sequencing uncovers genetic etiology in autosomal recessive nonsyndromic deafness in a large multiethnic cohort. Genet. Med. 18, 364-371. doi: 10.1038/gim.2015.89

Battelino, S., Klancar, G., Kovac, J., Battelino, T., and Trebusak Podkrajsek, K. (2015). TMPRSS3 mutations in autosomal recessive nonsyndromic hearing loss. Eur. Arch. Otorhinolaryngol. 273, 1151-1154. doi: 10.1007/s00405-0153671-0

Borck, G., Ur Rehman, A., Lee, K., Pogoda, H.-M., Kakar, N., von Ameln, S., et al. (2011). Loss-of-function mutations of ILDR1 cause autosomal-recessive hearing impairment DFNB42. Am. J. Hum. Genet. 88, 127-137. doi: 10.1016/j. ajhg.2010.12.011

Diaz-Horta, O., Duman, D., Foster, J., Sirmacı, A., Gonzalez, M., Mahdieh, N., et al. (2012). Whole-exome sequencing efficiently detects rare mutations in autosomal recessive nonsyndromic hearing loss. PLOS ONE 7:e50628. doi: 10.1371 /journal.pone.0050628

Higashi, T., Katsuno, T., Kitajiri, S.-I., and Furuse, M. (2015). Deficiency of angulin-2/ILDR1, a tricellular tight junction-associated membrane protein, causes deafness with cochlear hair cell degeneration in mice. PLoS ONE 10:e0120674. doi: 10.1371/journal.pone.0120674

Higashi, T., Tokuda, S., Kitajiri, S., Masuda, S., Nakamura, H., Oda, Y., et al. (2013). Analysis of the "angulin" proteins LSR, ILDR1 and ILDR2tricellulin recruitment, epithelial barrier function and implication in deafness pathogenesis. J. Cell Sci. 126, 966-977. doi: 10.1242/jcs.116442

Kim, N. K. D., Higashi, T., Lee, K. Y., Kim, A. R., Kitajiri, S., Kim, M. Y., et al. (2015). Downsloping high-frequency hearing loss due to inner ear tricellular tight junction disruption by a novel ILDR1 mutation in the Ig-like domain. PLoS ONE 10:e0116931. doi: 10.1371/journal.pone.0116931

Kovač, J., Macedoni Lukšič, M., Trebušak Podkrajšek, K., Klančar, G., and Battelino, T. (2014). Rare single nucleotide polymorphisms in the regulatory regions of the superoxide dismutase genes in autism spectrum disorder. Autism Res. 7, 138-144. doi: 10.1002/aur.1345 principle of genetic diagnosis where the most common causes of specific disease are tested first, the identification of rare, sporadic mutations in genes not typical for a particular population would require much more time and resources, influencing the genetic diagnosis outcome as well as the patient's quality of life.

\section{AUTHOR CONTRIBUTIONS}

GK prepared the NGS libraries and performed sequencing. KP performed validation of genetic results, supervised the genetic diagnostic procedures and contributed to the manuscript. SB performed clinical evaluation of the family, performed cochlear implant surgery and contributed to the manuscript. JK analyzed NGS data, performed Sanger sequencing and wrote the manuscript.

\section{FUNDING}

This work was supported by the Slovenian Research Agency (grants P3-0343, J3-6798, J3-2412 and J3-6800).

Lotti, M. T., Leipzig, J. N., Derbeneva, O., Xie, M. H., Chalkia, D., Sarmady, M., et al. (2013). mtDNA variation and analysis using mitomap and mitomaster. Curr. Protoc. Bioinformatics 1, 1.23.1-1.23.26. doi: 10.1002/0471250953. bi0123s44

Mehrjoo, Z., Babanejad, M., Kahrizi, K., and Najmabadi, H. (2015). Two novel mutations in ILDR1 gene cause autosomal recessive nonsyndromic hearing loss in consanguineous Iranian families. J. Genet. 94, 483-487. doi: 10.1007/s12041015-0537-6

Morozko, E. L., Nishio, A., Ingham, N. J., Chandra, R., Fitzgerald, T., Martelletti, E., et al. (2014). ILDR1 null mice, a model of human deafness DFNB42, show structural aberrations of tricellular tight junctions and degeneration of auditory hair cells. Hum. Mol. Genet. 24, 609-624. doi: 10.1093/hmg/ddu474

Pala, M., Olivieri, A., Achilli, A., Accetturo, M., Metsplau, E., Reidla, M., et al. (2012). Mitochondrial DNA signals of late glacial recolonization of europe from near eastern refugia. Am. J. Hum. Genet. 90, 915-924. doi: 10.1016/j.ajhg.2012. 04.003

Parker, M., and Bitner-Glindzicz, M. (2015). Genetic investigations in childhood deafness. Arch. Dis. Child 100, 271-278. doi: 10.1136/archdischild-2014-306099

Petersen, M. B., and Willems, P. J. (2006). Non-syndromic, autosomal-recessive deafness. Clin. Genet. 69, 371-392. doi: 10.1111/j.1399-0004.2006.00613.x

Ramzan, K., Taibah, K., Tahir, A. I., Al-Tassan, N., Berhan, A., Khater, A. M., et al. (2014). ILDR1: novel mutation and a rare cause of congenital deafness in the Saudi Arabian population. Eur. J. Med. Genet. 57, 253-258. doi: 10.1016/j.ejmg. 2014.04.004

Sang, Q., Li, W., Xu, Y., Qu, R., Xu, Z., Feng, R., et al. (2015). ILDR1 deficiency causes degeneration of cochlear outer hair cells and disrupts the structure of the organ of Corti: a mouse model for human DFNB42. Biol. Open 4, 411-418. doi: $10.1242 /$ bio. 201410876

Sang, Q., Zhang, J., Feng, R., Wang, X., Li, Q., Zhao, X., et al. (2014). Ildrlb is essential for semicircular canal development, migration of the posterior lateral line primordium and hearing ability in zebrafish: implications for a role in the recessive hearing impairment DFNB42. Hum. Mol. Genet. 23, 6201-6211. doi: $10.1093 / \mathrm{hmg} / \mathrm{ddu} 340$

Thorvaldsdóttir, H., Robinson, J. T., and Mesirov, J. P. (2013). Integrative Genomics Viewer (IGV): high-performance genomics data visualization and exploration. Brief. Bioinform. 14, 178-192. doi: 10.1093/bib/bbs017

Van Camp, G., and Smith, R. (2017). Hereditary Hearing Loss Homepage. Available at: http://hereditaryhearingloss.org [accessed May 11, 2017]. 
Yan, D., Tekin, M., Blanton, S. H., and Liu, X. Z. (2013). Next-generation sequencing in genetic hearing loss. Genet. Test. Mol. Biomark. 17, 581-587. doi: $10.1089 / \mathrm{gtmb} .2012 .0464$

Conflict of Interest Statement: The authors declare that the research was conducted in the absence of any commercial or financial relationships that could be construed as a potential conflict of interest.
Copyright (c) 2017 Kovač, Klančar, Trebušak Podkrajšek and Battelino. This is an open-access article distributed under the terms of the Creative Commons Attribution License (CC BY). The use, distribution or reproduction in other forums is permitted, provided the original author(s) or licensor are credited and that the original publication in this journal is cited, in accordance with accepted academic practice. No use, distribution or reproduction is permitted which does not comply with these terms. 